\title{
Resultados preliminares del estudio de la influencia de adición de harina de pórfido en la nutrición de un cultivo aplicado al caso de la lechuga (Lactuca Sativa L CV Neruda)
}

\author{
A. Fernández López ${ }^{1}$, M. Soler-Méndez², J.M. Molina-Martínez ${ }^{2}$, A. Ruiz-Canales ${ }^{1}$ \\ 1 Departamento de Ingeniería, Universidad Miguel Hernández de Elche, Escuela Politécnica Superior de \\ Orihuela (EPSO-UMH). Crtra. de Beniel, km 3,2. 03312 Orihuela (Alicante), Spain. \\ 2 Grupo de I+D+i de Ingeniería Agromótica y del Mar. UPCT. C/ del Ángel s/n. Ed. ELDI E1.06.30202 \\ Cartagena (Murcia), Spain
}

\begin{abstract}
Resumen: Se desarrolló un experimento en una parcela de la Escuela Politécnica Superior de Orihuela (EPSO) de la Universidad Miguel Hernández de Elche (UMH). En la citada parcela se aplicó una estrategia de riego común para mantener la tensión del suelo entre 10 y $20 \mathrm{mb}$. Se escogió como cultivo de control la lechuga (Lactuca Sativa L Neruda) pues tiene un ciclo corto y se obtienen resultados de una manera relativamente rápida y sencilla. Se aplicaron diferentes aportes nutricionales y de enmiendas (T1, T2, T3 y T4) durante tres campañas. Para el estudio nutricional de las hojas, a lo largo de todo el experimento, periódicamente se midieron periódicamente: cuatro análisis (uno por tratamiento) al final de la primera campaña; cuatro análisis (uno por tratamiento) al final de la segunda campaña; cuatro análisis (uno por tratamiento) al final de la tercera campaña. Los valores de peso de cosecha (peso por planta) mostraron que en la primera cosecha se obtuvo el mayor peso en el tratamiento T3, seguido del tratamiento T1, T2 y T4. Para la segunda cosecha, el mayor peso se dio en el tratamiento T3, seguido de T4, T2 y T1. Los valores de la segunda cosecha fueron un 3\% menores. En la tercera cosecha se obtuvieron mejores resultados de peso de cosecha en los tratamientos T1 y T2 respecto a T3 y T4. Ello indica que se produce homogeneización del estado vegetativo respecto al riego en la segunda campaña y tercera campañas, aunque los valores de cosecha han mostrado peores resultados en la segunda campaña. La influencia en los parámetros de cosecha se muestra en la tercera cosecha y posteriores (si se realizaran) ya que el suelo ha almacenado una serie de elementos y compuestos. El efecto nutricional favorable se presenta a partir de la tercera campaña.
\end{abstract}

Palabras clave: enmienda, gestión del agua y nutrientes en riego, análisis de hoja, riego localizado, horticultura

\section{Introducción}

La agricultura es el sector productivo que mayor cantidad de agua consume. De todo el agua dulce que alberga nuestro planeta, el $70 \%$ es destinada a la agricultura de regadío y se espera que para 2050 el consumo de agua para producir alimentos aumente un $60 \%$ [1]. Con el paso del tiempo está convirtiéndose en un recurso cada vez más escaso y costoso debido a ciertos inconvenientes como las pérdidas del agua consuntiva debido al exceso de riego [2]. Por otra parte, el aumento de la demanda, la contaminación de las aguas superficiales y subterráneas debido al uso de fertilizantes químicos y la histórica escasez de recursos hídricos que caracteriza 


\section{CONGRESO IBÉRICO DE AGROINGENIERÍA \\ X CONGRESSO IBÉRICO DE AGROENGENHARIA \\ 3 - 6 septiembre 2019, Huesca - España}

al sur de Europa debido a la aridez del clima y a las sequías, provoca que en esta zona el suministro de agua nunca esté garantizado, por lo que se hace necesario tomar medidas para utilizarla de un modo racional y eficiente [3].

La utilización de harina de roca de pórfido en sus dos variantes, con materia orgánica y sin materia orgánica, otorga al suelo una capacidad de retención de agua mayor frente a un suelo en el que no se ha utilizado ninguna enmienda. Esta capacidad de retención de agua es un $13 \%$ mayor con la utilización de la harina de roca de pórfido y un $15 \%$ en el caso de Bocashi. Se trata de una palabra de origen japonés que indica que es materia orgánica fermentada. Esta característica se traduce en una disminución del periodo de riego y por tanto un mejor aprovechamiento del agua y además un aporte de materia orgánica en el caso del Bocashi.

La adición de harina de roca de pórfido supone un aporte mineral al suelo debido a su composición química. Este aporte mineral puede suponer una mejora en la concentración de nutrientes disponibles para la planta en el suelo y este estudio trata de determinar de manera cuantitativa esta mejora y cuánto tiempo es necesario hasta que la planta puede aprovechar estos nutrientes. Se presenta en esta comunicación un ensayo con tratamiento diferencial de adición de diferentes proporciones de materia orgánica y enmienda (harina de pórfido) en suelo desnudo y para el cultivo de lechuga.

\section{Materiales y métodos}

Se desarrollaron los ensayos durante tres fechas (inicio: 10/11/16; mitad: 2/05/17; final: 19/04/18) en una parcela experimental de $300 \mathrm{~m}^{2}$ de la Escuela Politécnica Superior de Orihuela (EPSO) de la Universidad Miguel Hernández de Elche (UMH). En la citada parcela se instaló un sistema de riego localizado con cuatro sectores para aplicar cuatro tratamientos diferenciales respecto a las propiedades del suelo de cultivo. Cada tratamiento ocupaba una superficie de 75 $\mathrm{m}^{2}$, con un número de 225 lechugas por tratamiento. El desarrollo experimental principal de este proyecto fue el estudio del efecto en las propiedades texturales y edáficas del suelo, así como sus efectos en la producción hortícola, de la adición de harina de roca de pórfido, material desarrollado por la empresa Fulsan, S.A., a un suelo agrícola. Se escogió como cultivo de control la lechuga (Lactuca Sativa L Neruda) pues tiene un ciclo corto y se obtienen resultados de una manera relativamente rápida y sencilla. Se realizaron análisis periódicos de la composición química de las hojas y principales parámetros de cosecha (peso, producción por superficie, calibre, entre otros). En este caso se muestra solamente el análisis químico de las hojas y la producción obtenida en cada tratamiento.

Respecto a los tratamientos realizados se focalizó en la influencia de la adición de la harina de roca Fulsan en la producción agrícola. Se realizaron cuatro tratamientos en el suelo de la parcela experimental utilizada. Inicialmente se dividió la parcela en cuatro sectores de riego independientes. Se hizo el seguimiento del cultivo de lechuga durante tres campañas de riego.

En el primer tratamiento (T1) se le añadió al suelo al inicio de la campaña una cantidad convenida de harina de roca Fulsan y se aplicó junto con un abonado de fondo inorgánico. Durante las dos campañas de riego de los dos ciclos de cultivo se le aplicó al correspondiente sector (T1) de la plantación de lechuga, además de las correspondientes dosis de riego establecidas, un abonado inorgánico (fertirriego).

Para el segundo tratamiento (T2) se mezclaron harina de roca Fulsan y compost (Bocashi) con el suelo agrícola de la parcela experimental y esta mezcla constituyó un abonado de fondo orgánico. Durante las dos campañas de riego se le aplicó al correspondiente sector (T2) de la plantación de lechuga, además de las correspondientes dosis de riego establecidas, un fertirriego orgánico. El tratamiento T2 no recibió fertirriego inorgánico.

Para el tercer tratamiento (T3) no se añadió ninguna sustancia inicialmente al suelo agrícola. Durante las dos campañas de riego de los dos ciclos de cultivo se le aplicó al correspondiente sector 


\section{CONGRESO IBÉRICO DE AGROINGENIERÍA \\ X CONGRESSO IBÉRICO DE AGROENGENHARIA \\ 3 - 6 septiembre 2019, Huesca - España}

(T3) de la plantación de lechuga, además de las correspondientes dosis de riego establecidas, un abonado inorgánico (fertirriego), como en el tratamiento T1.

Adicionalmente se consideró incluir un cuarto tratamiento control (T4) para compararlo con los tratamientos anteriores. A este último tratamiento no se añadió abonado de fondo ni se le añadió fertirriego. Únicamente se le aplicó una dosis de riego similar a los tratamientos anteriores.

A los tratamientos T1 y T3 se les añadió un abonado de fondo inorgánico.

La dosificación del abonado de fondo y la adición de enmiendas fue similar en las dos campañas y se resume en lo siguiente.

Tratamiento $\mathrm{T} 1$.

- $70 \mathrm{Kg}$. Harina de pórfido por bancada. Total $210 \mathrm{Kg}$.

- 12,5 Kg. Yara Miila Complex (ARA) N-P-K. 12-11-18 más Boro, Hierro, Manganeso y Zinc.

- 12,5 Kg. Durasop Actibion N Plus (Fertinagro) N-P-K. 20-5-5, Mg-S. 2-15 y micronutrientes.

Tratamiento T 2.

- $170 \mathrm{Kg}$. Bocashi por bancada. Total $510 \mathrm{Kg}$.

Tratamiento T 3.

- 12,5 Kg. Yara Miila Complex (ARA) N-P-K- 12-11-18 más Boro, Hierro, Manganeso y Zinc.

- 12,5 Kg. Durasop Actibion N Plus (Fertinagro) N-P-K. 20-5-5, Mg-S. 2-15 y micronutrientes.

El plan de abonado inorgánico para los tratamientos T1 y T3 se estableció por la empresa suministradora del abono y se expresa en las Tablas 1 y 2.

Tabla 1. Plan de abonado en los tratamientos T1 y T3. $1^{\underline{o}}$ y $2^{\circ}$ Campaña.

Producción media (Tm/Ha)

Producción esperada ( $\mathrm{Tm} / \mathrm{Ha})$
40

40

\begin{tabular}{|c|c|c|c|c|c|c|c|c|}
\hline & & & & & SUM & $(\mathrm{Kg})$ & cela) & \\
\hline & & & & & QUाI & & & \\
\hline & & RIQUEZAS & 1 & 2 & 3 & 4 & 5 & 6 \\
\hline & SUMMUM & $12-40-6$ & - & - & - & - & - & - \\
\hline $\mathrm{L}_{3}$ & SUMMUM & $18-5-5$ & - & 0,80 & 2,50 & 2,60 & 2,60 & 2,60 \\
\hline 3 & SUMMUM & $12-5-40$ & - & - & - & - & - & - \\
\hline है & SUMMUM & $15-15-15$ & 0,80 & 1,60 & 3,30 & 4,30 & 4,30 & 4,30 \\
\hline & & & - & - & - & - & - & - \\
\hline
\end{tabular}

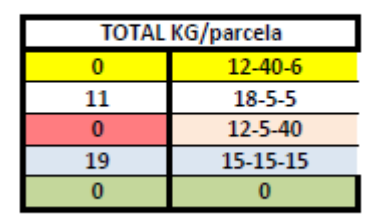

\begin{tabular}{c|c|c|c|c|c|c|c|}
\multicolumn{1}{c}{} & \multicolumn{8}{c}{ UNIDADES FERTILIZANTES APORTADAS (U.F./Ha) } \\
\cline { 3 - 9 } & NECESIDADES & 1 & 2 & 3 & 4 & 5 & 6 \\
\hline N & 150 & 0,16 & 0,32 & 0,66 & 0,86 & 0,86 & 0,86 \\
\hline P205 & 110 & - & 0,08 & 0,25 & 0,26 & 0,26 & 0,26 \\
\cline { 3 - 9 } K20 & 100 & - & 0,08 & 0,25 & 0,26 & 0,26 & 0,26 \\
\hline
\end{tabular}

\begin{tabular}{|c|c|}
\hline TOTAL U.F.APORTADAS \\
\hline 4 & $\mathrm{~N}$ \\
\hline 1 & $\mathrm{P} 205$ \\
\hline 1 & $\mathrm{~K} 20$ \\
\hline $\mathrm{Si}$ & $\mathrm{Mn}$ \\
\hline $\mathrm{Si}$ & $\mathrm{Cu}$ \\
\hline $\mathrm{Si}$ & $\mathrm{Zn}$ \\
\hline
\end{tabular}

Tabla 2. Plan de abonado en los tratamientos T1 y T3. $3^{\circ}$ Campaña.

\begin{tabular}{|l|c|c|c|c|c|c|c|c|c|c|c|c|}
\cline { 2 - 14 } & \multicolumn{4}{|c|}{ Aniones mMol/l } & \multicolumn{4}{c|}{ Cationes mMol/l } & & $\mathrm{CE}$ \\
\cline { 2 - 13 } & $\mathrm{NO}_{3}{ }^{--}$ & $\mathrm{N}_{2} \mathrm{PO}_{4}^{-}$ & $\mathrm{SO}_{4}^{--}$ & $\mathrm{HCO}_{3}^{-}$ & $\mathrm{Cl}^{-}$ & $\mathrm{NH}_{4}^{+}$ & $\mathrm{K}^{+}$ & $\mathrm{Ca}^{++}$ & $\mathrm{Mg}^{++}$ & $\mathrm{Na}^{+}$ & $\mathrm{Ph}$ & $\mathrm{mS} / \mathrm{cm}$ \\
\hline Agua de riego & - & - & 4 & 3 & 7 & - & 0,28 & 3 & 2 & 8,26 & 8,2 & 1,67 \\
\hline Solución ideal & 9,12 & 1,6 & - & 0,5 & - & 0,5 & 6 & 4 & 2 & - & 5,8 & 2,8 \\
\hline Aportes previstos & 9,12 & 1,6 & - & $-2,6$ & - & 0,5 & 5,72 & 1 & - & - & 5,8 & 2,8 \\
\hline
\end{tabular}


X CONGRESO IBÉRICO DE AGROINGENIERÍA

X CONGRESSO IBÉRICO DE AGROENGENHARIA

3 - 6 septiembre 2019, Huesca - España

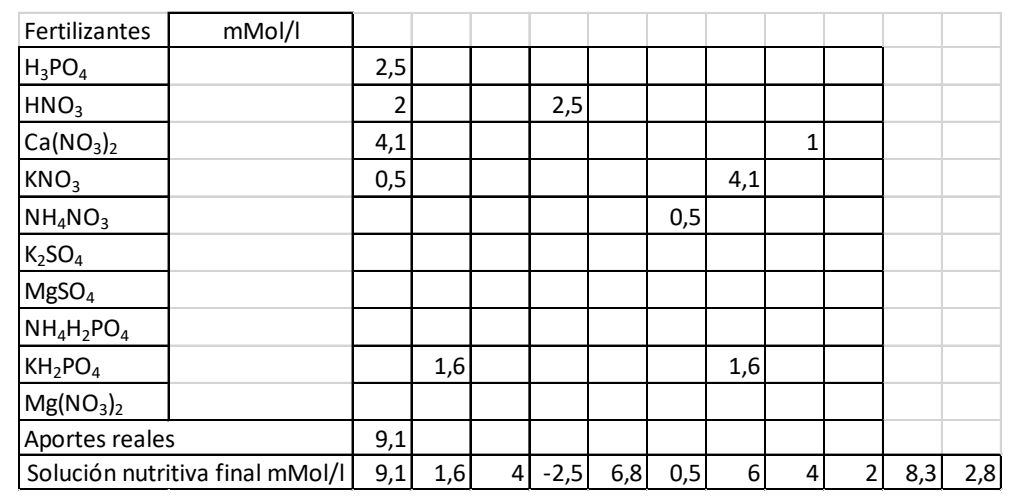

\begin{tabular}{|c|c|c|c|c|c|c|}
\hline & Fertilizantes par & $\mathrm{Id}$ & $100 \mathrm{vec}$ & entrada & 5001 & 2501 \\
\hline Acido nítrico (37\%) & & $\mathrm{x}$ & $13,8=$ & & & \\
\hline Acido nítrico (59\%) & 2,5 & $\mathrm{x}$ & $7,8=$ & $19,5 \mathrm{I}$ & 9,751 & 4,81 \\
\hline Acido fosfórico (37\%) & & $x$ & $21,2=$ & & & \\
\hline Acido fosfórico $(75 \%)$ & & $\mathrm{x}$ & $8,2=$ & & & \\
\hline Nitrato potásico & 4,12 & $\mathrm{x}$ & $10,1=$ & $41,6 \mathrm{~kg}$ & $20,8 \mathrm{~kg}$ & $10,4 \mathrm{~kg}$ \\
\hline Nitrato calcico & 1 & $\mathrm{x}$ & $18,1=$ & 18,1 & 9,05 & 4,5 \\
\hline Nitrato amónico & 0,5 & $\mathrm{x}$ & $8=$ & $4 \mathrm{~kg}$ & $2 \mathrm{~kg}$ & $1 \mathrm{~kg}$ \\
\hline Sulfato potásico & & $\mathrm{x}$ & $17,4=$ & & & \\
\hline Sulfato magnésico & & $x$ & $24,6=$ & & & \\
\hline Fosfato monoamónico & & $x$ & $11,5=$ & & & \\
\hline Fosfato monopotásico & 1,6 & $\mathrm{x}$ & $13,6=$ & $21,76 \mathrm{~kg}$ & $10,88 \mathrm{~kg}$ & $5,44 \mathrm{~kg}$ \\
\hline Nitrato Magnésico & & $\mathrm{x}$ & $25,6=$ & & & \\
\hline \multicolumn{7}{|c|}{ Complejo de Microelementos } \\
\hline \multicolumn{4}{|l|}{$\mathrm{Fe}^{++}$} & $2,083 \mathrm{~kg}$ & & \\
\hline
\end{tabular}

En el tratamiento T2 únicamente se aportó un abono orgánico con una cadencia de dos veces por semana a lo largo de todo el período experimental. Este tratamiento lo dosificó la empresa Fulsan, S.A.

Para el estudio nutricional de las hojas, a lo largo de todo el experimento, se midieron periódicamente:

Cuatro análisis (uno por tratamiento) al final de la primera campaña.

Cuatro análisis (uno por tratamiento) al final de la segunda campaña.

Los resultados de los análisis se muestran en las tablas 3, 4,5 y 6 .

Tabla 3. Composición nutricional de la hoja para el tratamiento T1 a lo largo del experimento.

\begin{tabular}{cccccc}
\hline & $\begin{array}{c}\text { Valores } \\
\text { Normales }\end{array}$ & $\begin{array}{c}\text { Valores } \\
\text { Deficientes }\end{array}$ & Campaña 1 & Campaña 2 & Campaña 3 \\
\hline Sodio \% & $<0.30 \%$ & - & 0,44 & 0,23 & 0,49 \\
Potasio \% & $4.5-6.3 \%$ & $<2.5 \%$ & 5,9 & 4,1 & 4,12 \\
Calcio \% & $0.50-0.75 \%$ & $<0.28 \%$ & 1,0 & 0,39 & 0,41 \\
Magnesio \% & $0.25-0.35 \%$ & $<0.20 \%$ & 0,32 & 0,19 & 0,27 \\
Hierro ppm & $>75 \mathrm{mgkg}-1$ & $<50 \mathrm{mgkg}-1$ & 100,4 & 57,6 & 82,5 \\
Cobre ppm & $50 \mathrm{mgkg}-1$ & $<2.0 \mathrm{mgkg}-1$ & 24,6 & 9,6 & 16,9 \\
Manganeso & $>5.0 \mathrm{mgkg}-$ & $<20 \mathrm{mgkg}-1$ & 35,3 & 15,6 & 17,1 \\
ppm & 1 & & & & \\
Zinc ppm & $>25 \mathrm{mgkg}-1$ & $<22 \mathrm{mgkg}-1$ & 134,2 & 51,4 & 55,2 \\
Nitrógeno & $3.5-5.0 \%$ & $<2.5 \%$ & 3,2 & 3,4 & 2,0 \\
kjeldahl \% & & & & & \\
Fósforo \% & $0.30-0.60 \%$ & $<0.20 \%$ & 0,69 & 0,46 & 0,59 \\
Boro ppm & $>25 \mathrm{mgkg}-1$ & $<20 \mathrm{mgkg}-1$ & 37,5 & 18,4 & 12,1 \\
\hline
\end{tabular}




\section{CONGRESO IBÉRICO DE AGROINGENIERÍA \\ X CONGRESSO IBÉRICO DE AGROENGENHARIA \\ 3-6 septiembre 2019, Huesca - España}

Tabla 4. Composición nutricional de la hoja para el tratamiento T2 a lo largo del experimento.

\begin{tabular}{cccccc}
\hline & $\begin{array}{c}\text { Valores } \\
\text { Normales }\end{array}$ & $\begin{array}{c}\text { Valores } \\
\text { Deficientes }\end{array}$ & Campaña 1 & Campaña 2 & Campaña 3 \\
\hline Sodio $\%$ & $<0.30 \%$ & - & 0,41 & 0,22 & 0,46 \\
Potasio \% & $4.5-6.3 \%$ & $<2.5 \%$ & 6,1 & 4,2 & 4,42 \\
Calcio \% & $0.50-0.75 \%$ & $<0.28 \%$ & 0,85 & 0,60 & 0,52 \\
Magnesio \% & $0.25-0.35 \%$ & $<0.20 \%$ & 0,29 & 0,25 & 0,29 \\
Hierro ppm & $>75 \mathrm{mgkg}-1$ & $<50 \mathrm{mgkg}-1$ & 83,2 & 68,1 & 101,3 \\
Cobre ppm & 50 mgkg-1 & $<2.0 \mathrm{mgkg}-1$ & 25,1 & 6,7 & 17,5 \\
Manganeso & $>5.0 \mathrm{mgkg}-$ & $<20 \mathrm{mgkg}-1$ & 36,7 & 21,9 & 24,3 \\
ppm & 1 & & & & \\
Zinc ppm & $>25 \mathrm{mgkg}-1$ & $<22$ mgkg-1 & 60,6 & 41,8 & 56,7 \\
Nitrógeno & $3.5-5.0 \%$ & $<2.5 \%$ & 3,4 & 3,2 & 4,6 \\
kjeldahl \% & & & & & \\
Fósforo $\%$ & $0.30-0.60 \%$ & $<0.20 \%$ & 0,72 & 0,49 & 0,78 \\
Boro ppm & $>25 \mathrm{mgkg}-1$ & $<20 \mathrm{mgkg}-1$ & 32,2 & 19,2 & 19,7 \\
\hline
\end{tabular}

Tabla 5. Composición nutricional de la hoja para el tratamiento T3 a lo largo del experimento.

\begin{tabular}{cccccc}
\hline & $\begin{array}{c}\text { Valores } \\
\text { Normales }\end{array}$ & $\begin{array}{c}\text { Valores } \\
\text { Deficientes }\end{array}$ & Campaña 1 & Campaña 2 & Campaña 3 \\
\hline Sodio \% & $<0.30 \%$ & - & 0,31 & 0,21 & 0,46 \\
Potasio \% & $4.5-6.3 \%$ & $<2.5 \%$ & 5,1 & 3,5 & 3,61 \\
Calcio \% & $0.50-0.75 \%$ & $<0.28 \%$ & 0,41 & 0,36 & 0,35 \\
Magnesio \% & $0.25-0.35 \%$ & $<0.20 \%$ & 0,33 & 0,18 & 0,21 \\
Hierro ppm & $>75 \mathrm{mgkg}-1$ & $<50 \mathrm{mgkg}-1$ & 86,6 & 66,3 & 74,3 \\
Cobre ppm & $50 \mathrm{mgkg}-1$ & $<2.0 \mathrm{mgkg}-1$ & 40,8 & 7,9 & 16,3 \\
Manganeso & $>5.0 \mathrm{mgkg}-$ & $<20$ mgkg-1 & 38,9 & 15,1 & 14,3 \\
ppm & 1 & & & & \\
Zinc ppm & $>25 \mathrm{mgkg}-1$ & $<22$ mgkg-1 & 43,5 & 49,4 & 43,7 \\
Nitrógeno & $3.5-5.0 \%$ & $<2.5 \%$ & 3,8 & 3,6 & 3,6 \\
kjeldahl \% & & & & & \\
Fósforo \% & $0.30-0.60 \%$ & $<0.20 \%$ & 0,42 & 0,44 & 0,59 \\
Boro ppm & $>25 \mathrm{mgkg}-1$ & $<20 \mathrm{mgkg}-1$ & 28,9 & 17,4 & 13 \\
\hline
\end{tabular}

Tabla 6. Composición nutricional de la hoja para el tratamiento T4 a lo largo del experimento.

\begin{tabular}{cccccc}
\hline & $\begin{array}{c}\text { Valores } \\
\text { Normales }\end{array}$ & $\begin{array}{c}\text { Valores } \\
\text { Deficientes }\end{array}$ & Campaña 1 & Campaña 2 & Campaña 3 \\
\hline Sodio \% & $<0.30 \%$ & - & 0,43 & 0,21 & 0,35 \\
Potasio \% & $4.5-6.3 \%$ & $<2.5 \%$ & 6,0 & 4,4 & 3,41 \\
Calcio \% & $0.50-0.75 \%$ & $<0.28 \%$ & 0,99 & 0,52 & 0,35 \\
Magnesio \% & $0.25-0.35 \%$ & $<0.20 \%$ & 0,34 & 0,21 & 0,21 \\
Hierro ppm & $>75 \mathrm{mgkg}-1$ & $<50 \mathrm{mgkg}-1$ & 89,8 & 57,7 & 70,7 \\
Cobre ppm & $50 \mathrm{mgkg}-1$ & $<2.0 \mathrm{mgkg}-1$ & 26,9 & 8,1 & 15,3 \\
Manganeso & $>5.0 \mathrm{mgkg}-1$ & $<20 \mathrm{mgkg}-1$ & 40,2 & 19,7 & 16,7 \\
ppm & & & & & \\
Zinc ppm & $>25 \mathrm{mgkg}-1$ & $<22 \mathrm{mgkg}-1$ & 55,7 & 40,2 & 45,1 \\
Nitrógeno & $3.5-5.0 \%$ & $<2.5 \%$ & 4,2 & 2,9 & 3,1 \\
kjeldahl \% & & & & & \\
Fósforo \% & $0.30-0.60 \%$ & $<0.20 \%$ & 0,58 & 0,38 & 0,57 \\
Boro ppm & $>25 \mathrm{mgkg}-1$ & $<20 \mathrm{mgkg}-1$ & 23,2 & 22,7 & 9,7 \\
\hline
\end{tabular}




\section{CONGRESO IBÉRICO DE AGROINGENIERÍA \\ X CONGRESSO IBÉRICO DE AGROENGENHARIA \\ 3- 6 septiembre 2019, Huesca - España}

\section{Resultados y discusión}

El peso medio de la cosecha (por planta) para la primera campaña fue el siguiente:

Tratamiento T1: $1,195 \mathrm{~kg} \pm 0,303$

Tratamiento T2: $1,014 \mathrm{~kg} \pm 0,150$

Tratamiento T3: $1,249 \mathrm{~kg} \pm 0,259$

Tratamiento T4: $1,077 \mathrm{~kg} \pm 0,172$

El peso medio de la cosecha para la segunda campaña fue el siguiente:

Tratamiento T1: $0,758 \mathrm{~kg} \pm 0,192$

Tratamiento T2: $0,788 \mathrm{~kg} \pm 0,196$

Tratamiento T3: $1,076 \mathrm{~kg} \pm 0,222$

Tratamiento T4: $0,896 \mathrm{~kg} \pm 0,199$

Según lo expuesto, la producción en la segunda campaña fue de menor cuantía, alrededor de un $3 \%$ menor.

El peso medio de la cosecha para la tercera campaña fue el siguiente:

Tratamiento T1: $0,759 \mathrm{~kg} \pm 0,132$

Tratamiento T2: $1,251 \mathrm{~kg} \pm 0,157$

Tratamiento T3: $1,193 \mathrm{~kg} \pm 0,169$

Tratamiento T4: $1,017 \mathrm{~kg} \pm 0,117$

El peso medio de la cosecha fue mayor en el tratamiento T2 durante la tercera campaña. Se muestra la tendencia en esta última campaña. Se intuye que las propiedades de mejora que puede aportar la harina de pórfido relacionadas con el almacenamiento de agua y la posibilidad de suministro de nutrientes, se ha materializado en la tercera campaña.

Los valores de análisis de hoja muestran lo siguiente:

Sodio: En el tratamiento T4 se mejora la concentración de sodio en hoja en la segunda campaña desde carencia a valores normales, así como en T1, T2 y T3. Tienen valores similares. No hay una clara influencia de la adición de harina de pórfido en el estado nutricional de la hoja respecto al sodio.

Potasio: Los valores para el T4 han estado dentro de la normalidad y se pasa a una cierta carencia, pero sin ser excesiva, al igual que T2, T3 y T4. Los niveles son mayores en T2, seguidos de T4, T1 y T3. La harina de pórfido puede tener una cierta influencia en el estado nutricional del potasio en hoja, aunque no es muy significativo.

Calcio: Se han mantenido concentraciones normales. Son mayores en el T2 y T1. Puede tener una cierta influencia la adición de harina de pórfido en el estado nutricional del calcio en hoja.

Magnesio: Las lechugas han pasado de niveles normales a valores mínimos, pero sin ser carentes. La influencia de la adición de harina de pórfido es mayor en el T2 que en el resto.

Hierro: Se ha reducido en un $25 \%$ la concentración en el T4. Aunque no provoca carencias sí pueden producirse a medio plazo. Igual que en el resto. No ha habido una diferencia al aplicar harina de pórfido.

Cobre: Ha disminuido en el experimento y la dificultad de absorción puede ser problemática a la larga, aunque no llega a presentar carencia. No hay influencia en la adición de harina de pórfido.

Manganeso: El manganeso en el T4 se ha reducido a bajos niveles, pero no de carencia. Valores por debajo de T1, T2 y T3. La adición de harina de pórfido sí puede tener una influencia en la composición de la hoja.

Zinc: El zinc se ha mantenido en valores normales durante todo el experimento, como en T1, T2 y T3. Ha sido mayor en T1 y T2. Tiene una influencia clara la adición de harina de pórfido al estado nutricional de la hoja.

Nitrógeno: La concentración de este elemento se mantiene en la normalidad. Aunque el valor del porcentaje de nitrógeno ha sido inferior a los niveles óptimos para lechugas de 45 días (entre 3,5 y $5 \%$ ) en ningún momento del experimento se alcanzan valores que provoquen carencias en 


\section{CONGRESO IBÉRICO DE AGROINGENIERÍA \\ X CONGRESSO IBÉRICO DE AGROENGENHARIA \\ 3 - 6 septiembre 2019, Huesca - España}

el tratamiento T4. Similar al T1 y T3. La adición de harina de roca ha influido en estados nutricionales de menor cuantía que los tratamientos control. Hay que probarlo en nuevas campañas.

Fósforo: Los valores de la concentración de fósforo en el tratamiento T4 quedaron dentro del rango de los valores normales, igual que en el tratamiento T1, T2 y T3. Las mayores concentraciones se dieron en T1, seguido de T2. Se nota una cierta influencia en el estado nutricional.

Boro: El boro se ha visto reducido en las dos campañas para el T4. Se presentan más carencias que en T1, T2 y T3. La adición de harina de roca puede influir positivamente en el estado nutricional en hoja, en el tratamiento $\mathrm{T} 1$.

\section{Conclusiones}

Los valores de peso de cosecha (peso por planta) mostraron que en la primera cosecha se obtuvo el mayor peso en el tratamiento T3, seguido del tratamiento T1, T2 y T4.

Para la segunda cosecha, el mayor peso se dio en el tratamiento T3, seguido de T4, T2 y T1. Los valores de la segunda cosecha fueron un $3 \%$ menores.

Hay una cierta estabilización de la homogeneización del estado vegetativo respecto al riego en la segunda campaña, aunque los valores de cosecha han mostrado peores resultados. Puede que la influencia en los parámetros de cosecha se muestra en las siguientes cosechas ya que el suelo ha almacenado una serie de elementos y compuestos.

\section{Agradecimientos}

Los autores del artículo expresan su agradecimiento a la empresa FULSAN, S.A. por su apoyo económico. Igualmente se muestran agradecidos a la empresa TELENATURA EBT, S.L. y Viveros Simón Cases, S.L. por su apoyo tecnológico y agronómico.

\section{Referencias}

1. Yildiz, I. Review of climate change issues: A forcing function perspective in agricultural and energy innovation. International journal of energy research. 2019, 43, (2200-2215).

2. Wei, X.P., Xie, D.D., Mao, L.C., Xu, C.J., Luo, Z.S., Xia, M., Zhao, X.X., Han, X.Y., Lu, W.J. Excess water loss induced by simulated transport vibration in postharvest kiwifruit. Scientia Horticulturae. 2019, 250, (113-120).

3. Launay, M., Dugue, V., Faure, J.B., Coquery, M., Camenen, B., Le Coz, J. Numerical modelling of the suspended particulate matter dynamics in a regulated river network. Science of the total environment, 2019, 665, (591-605). 